\title{
Segmentation of Text and Graphics/Images Using the Gray-Level Histogram Fourier Transform
}

\author{
M.A. Patricio ${ }^{1}$, D. Maravall ${ }^{2}$ \\ ${ }^{1}$ Centro de Cálculo \\ ${ }^{2}$ Departamento de Inteligencia Artificial \\ Universidad Politécnica de Madrid \\ dmaravall@fi.upm.es
}

\begin{abstract}
One crucial issue in automatic document analysis is the discrimination between text and graphics/images. This paper presents a novel, robust method for the segmentation of text and graphics/images in digitized documents. This method is based on the representation of window-like portions of a document by means of their gray level histograms. Through empirical evidence it is shown that text and graphics/images regions have different gray level histograms. Unlike the usual approach for the characterization of histograms that is based on statistics parameters a novel approach is introduced. This approach works with the histogram Fourier transform since it possesses all the information contained in the histogram pattern. The next and logical step is to automatically select the most discriminant spectral components as far as the text and graphics/images segmentation goal is concerned. A fully automated procedure for the optimal selection of the discriminant features is also expounded. Finally, empirical results obtained for the text and graphics/images segmentation using a simple three-layer perceptron-like neural network are also discussed.
\end{abstract}

Keywords: Feature extraction and selection; Image analysis; Applications: automatic document analysis.

\section{Introduction - The Gray Level Histogram as a Discriminant Tool for Text and Graphics/Images Segmentation}

Document image analysis is an active research and development field [1] in which pattern recognition techniques are of the greatest interest. One critical issue in the automatic analysis of digitized documents is the separation of text and graphics/images. The text regions of the document are usually analyzed by means of well-known OCR techniques, whereas the graphics and images are just codified in order to obtain optimal storage and retrieval of such information. This communication describes a novel method for the segmentation of text and graphics/images. This method exploits the empirical evidence that regions of text and regions of graphics/images have very different gray level histograms. As an illustration, figure 1 shows two examples. Notice the application of a window on the original digitized document in order to compute the brightness histogram in small portions of the whole document. The practical issues concerning the window size and the scanning process 
over the complete document, although important, are not specifically considered in this paper.

\section{través de Internet de form: \\ S-cecureWay Software. Sun}
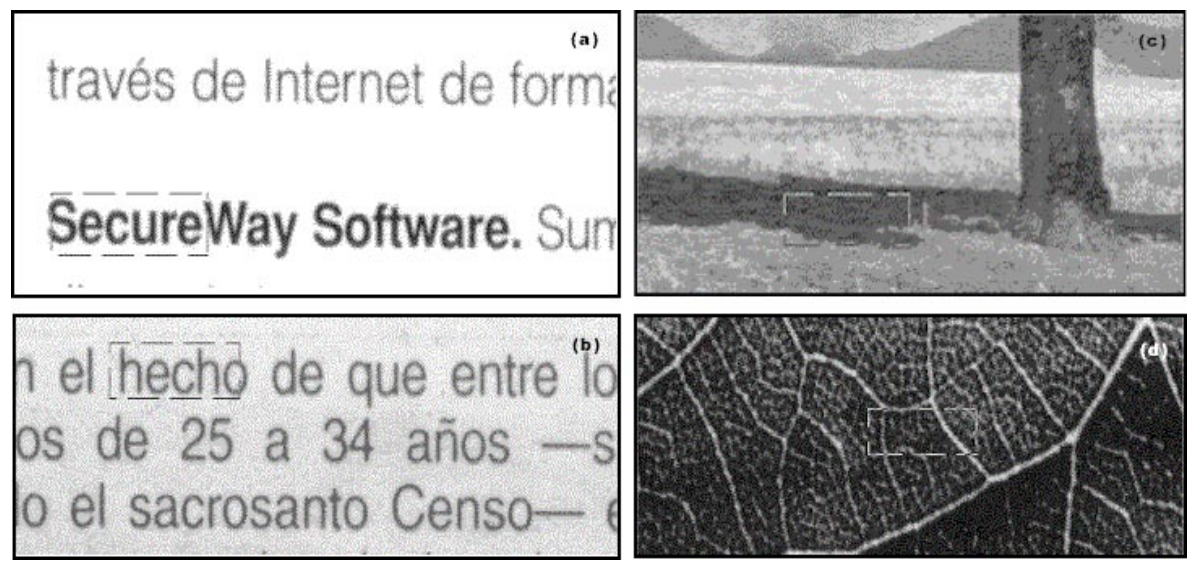

Fig. 1. Examples of text and images regions in digitized documents

In figure 2 the corresponding histograms are displayed. Notice the similarity between the text regions histograms and their differences with the image regions histograms. Therefore it seems reasonable to exploit the gray level histogram patterns for the discrimination between text and graphics/images.

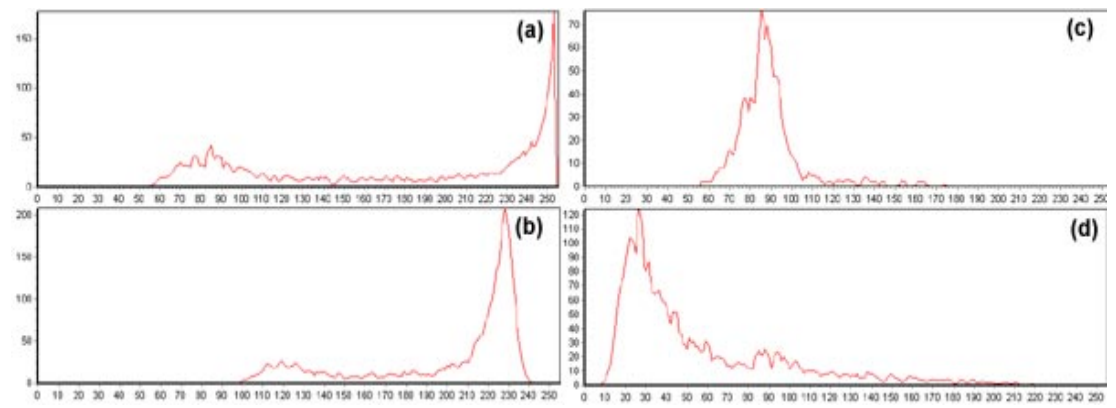

Fig. 2. Gray level histograms for the window indicated in figure 1.

After an exhaustive empirical work we have confirmed the similarity of the gray level histograms of text regions in which there appear a large accumulation of gray levels on the right side, corresponding to the background pixels and a small distribution on the low gray levels due to the characters' pixels. Almost all these histograms are purely bimodal and close to normal distributions. On the contrary, the graphics/images regions do not present such uniform pattern, which is logical due to the variety of the graphics and in particular of the images. Nevertheless, as far as the segmentation of text and graphics/images is concerned, the important issue is the uniformity of text histograms. 


\section{Histogram Fourier Transform}

Once the idea of using the histogram pattern as the basis for the segmentation of text and graphics/images is accepted, the next step is to obtain an appropriate set of discriminant features from the histogram. The usual approach is to compute firstorder statistics parameters: mean, variance, skewness, kurtosis, entropy, etc. Unfortunately, in many cases these discriminant features are not satisfactory as they do not convey all the discriminant information of the whole histogram and therefore some authors prefer to work with the complete gray level distributions [5]-[8].

In this paper we have taken an intermediate approach by using the magnitude spectrum of the histogram as the initial set of discriminant features.

Let $h(i)$ be the gray level histogram of some generic region - the scanning window - , then its Fourier transform is:

$$
\begin{gathered}
H(u)=\frac{1}{N} \sum_{i=0}^{N-1} h(i) e^{-j \frac{2 \pi}{N} i u} \\
0 \leq i \leq N-1 \quad ; \quad 0 \leq u \leq N-1
\end{gathered}
$$

The amplitude spectrum of $H(u)$ is the magnitude of $H(u)$ :

$$
\begin{gathered}
A(u)=\sqrt{H_{\mathrm{Re}}^{2}(u)+H_{\mathrm{Im}}^{2}(u)} \\
0 \leq u \leq N-1
\end{gathered}
$$

and its phase is:

$$
\begin{gathered}
\Psi(u)=\arctan \left[\frac{H_{\mathrm{Im}}(u)}{H_{\mathrm{Re}}(u)}\right] \\
0 \leq u \leq N-1
\end{gathered}
$$

For the characterization of the histogram pattern it is sufficient to concentrate on the magnitude of the Fourier transform and ignore the phase. Furthermore, the amplitude spectrum is invariant under gray level histogram translations:

$$
\text { If } h(i)=g(i-t), \forall i \in[0, N-1] \Rightarrow A\{H(u)\}=A\{G(u)\}, \forall u \in[0, N-1]
$$

\section{Automatic Selection of the Optimal Discriminant Features}

Although independent to translations, the amplitude spectrum has as many components as the original histogram, so that a drastic reduction in its dimension is crucial. Obviously, such reduction is aimed at obtaining the minimum number of the best discriminant features. In the next section we describe an automatic procedure for the selection of the best discriminant features of the amplitude spectrum. 


\section{Step 1. Data Normalization}

First, a zero mean and unit variance data normalization is applied to all of the magnitude spectrum components.

For a generic class or pattern, $\alpha_{\mathrm{k}}$, the $\mathrm{j}^{\text {th }}$ feature average, $\mathrm{m}_{\mathrm{j}}$, and $\mathrm{j}^{\text {th }}$ variance $\sigma_{\mathrm{j}}^{2}$, are computed as the sample mean and the sample variance:

$$
\begin{gathered}
\hat{\mathrm{m}}_{j}=\frac{1}{N_{j}} \sum_{i=1}^{N_{j}} x_{i j} \\
\hat{\sigma}_{j}^{2}=\frac{1}{N_{j}-1} \sum_{i=1}^{N_{j}}\left(x_{i j}-\hat{\mathrm{m}}_{j}\right)^{2}
\end{gathered}
$$

where $\mathrm{N}_{\mathrm{j}}$ is the number of the design samples available from class $\alpha_{\mathrm{k}}$.

Then, the generic $\mathrm{j}^{\text {th }}$ feature component, $\mathrm{x}_{\mathrm{j}}$, is normalized as follows:

$$
x_{j}^{*}=\frac{x_{j}-m_{j}}{\sigma_{j}}
$$

For the purpose of simplicity of notation we drop out the use of asterisks in the next section, although all the data have been previously normalized.

\section{Step 2. Discriminant Feature Ordering}

For each individual discriminant variable, $\mathrm{x}_{\mathrm{i}}$, the generalized Fisher ratio is computed:

$$
\begin{aligned}
& R_{i}=\frac{\frac{1}{N} \sum_{j=1}^{N}\left(m_{i j}-m_{i}\right)^{2}}{\sigma_{1}^{2}+\sigma_{2}^{2}+\ldots+\sigma_{N}^{2}} \\
& m_{i}=\frac{m_{i 1}+m_{i 2}+\ldots+m_{i N}}{N}
\end{aligned}
$$

where the index $\mathrm{j}=1,2, \ldots, \mathrm{N}$ represents the corresponding pattern or class. For the text and graphics/image segmentation case, obviously $\mathrm{j}=1,2$. The index $\mathrm{i}=1,2, \ldots, \mathrm{n}$ stands for the individual features and for digitized documents with 256 brightness values $i=1,2, \ldots, 256$.

After computing the Fisher ratios the features are arranged as follows:

$$
x_{1}, x_{2}, \ldots, x_{n} \quad / \quad R_{1}>R_{2}>\ldots>R_{N}
$$




\section{Step 3. Selection of the Feature Vector}

After completing step 2, the first action is to select the optimal individual feature. Let $\mathrm{x}_{1}$ be the most discriminant feature: i. e. $\mathrm{R}_{1}$ is the highest Fisher ratio in expression (8). Once the optimal feature $x_{1}$ has been chosen, the next action is to form all possible two-dimensional vectors that include the optimal feature $\mathrm{x}_{1}$ and to select the pair with the highest discriminant ratio. This process is repeated with three, four, five, etc components of the feature set until the performance improvement is less than certain threshold.

For the evaluation of the discriminant capacity of multidimensional feature vectors several indices or ratios can be used:

$$
\begin{aligned}
& J_{1}=\frac{\operatorname{trace}\left(S_{B}\right)}{\operatorname{trace}\left(S_{W}\right)} \\
& J_{2}=\frac{\operatorname{det}\left|S_{B}\right|}{\operatorname{det}\left|S_{W}\right|} \\
& J_{3}=\operatorname{trace}\left(S_{W}^{-1} S_{B}\right)
\end{aligned}
$$

$\mathrm{S}_{\mathrm{B}}$ is the between scatter matrix that provides a quantitative indication of the dispersion among the classes:

$$
S_{B}=\sum_{i=1}^{N} p_{i}\left(\mathbf{m}_{i}-\mathbf{m}\right)\left(\mathbf{m}_{i}-\mathbf{m}\right)^{T}
$$

where $\mathrm{p}_{\mathrm{i}}$ is the a priori probability of class $\alpha_{\mathrm{i}} ; \mathbf{m}_{\mathrm{i}}$ is the vector mean of the class $\alpha_{\mathrm{i}}$ and $\mathbf{m}$ is the global vector mean:

$$
\mathbf{m}=\sum_{i=1}^{N} p_{i} \mathbf{m}_{i}
$$

The within matrix, $\mathrm{S}_{\mathrm{W}}$, is the average covariance matrix:

$$
\begin{gathered}
S_{W}=\sum_{i=1}^{N} p_{i} \mathbf{C}_{i} \\
\mathbf{C}_{i}=E\left\{\left(\mathbf{x}-\mathbf{m}_{i}\right)\left(\mathbf{x}-\mathbf{m}_{i}\right)^{T}\right\}
\end{gathered}
$$

In figure 3 the flowchart of the automatic selection algorithm is displayed. 


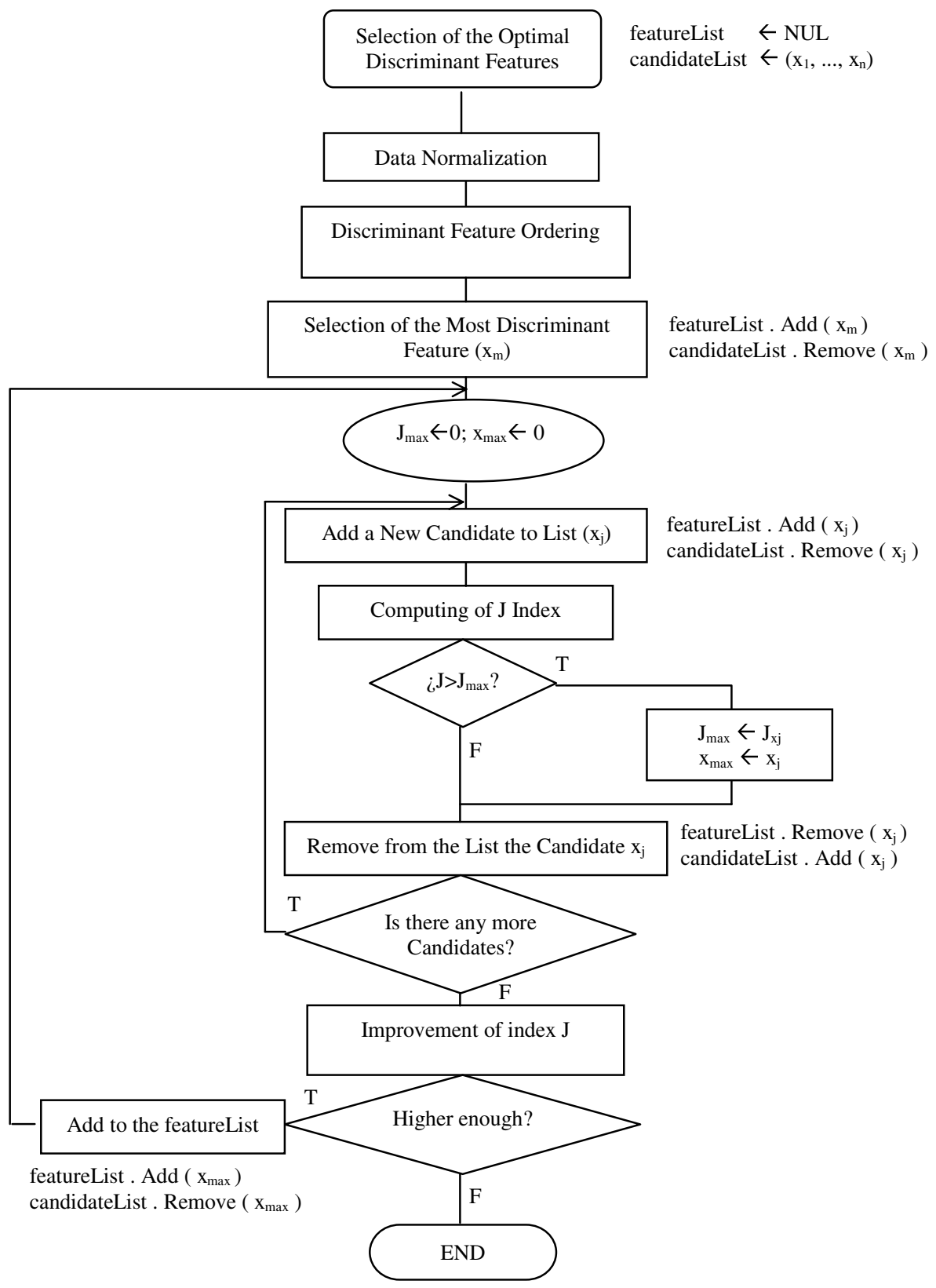

Fig. 3. Flowchart of the automatic feature selection algorithm. 


\section{Results}

Using a commercial 300 dpi scanner many different types of documents have been digitized in order to test the segmentation method. We have been careful in testing a large variety of fonts and types of documents: books, magazines, newspapers, etc. After empirical evaluation the final window size is $80 \times 35$ pixels. We have used 585 labeled samples for the automatic selection of the optimal set of discriminant features. After applying the method previously described, the following discriminant features were obtained at each sequential level:

$$
\begin{aligned}
& \text { Level 1 } \rightarrow\left(x_{161}\right) \\
& \text { Level 2 } \rightarrow\left(x_{161}, x_{47}\right) \\
& \text { Level 3 } \rightarrow\left(x_{161}, x_{47}, x_{209}\right)
\end{aligned}
$$

The algorithm ended at level 3 because the next level produced less than $1 \%$ improvement on the discriminant index.

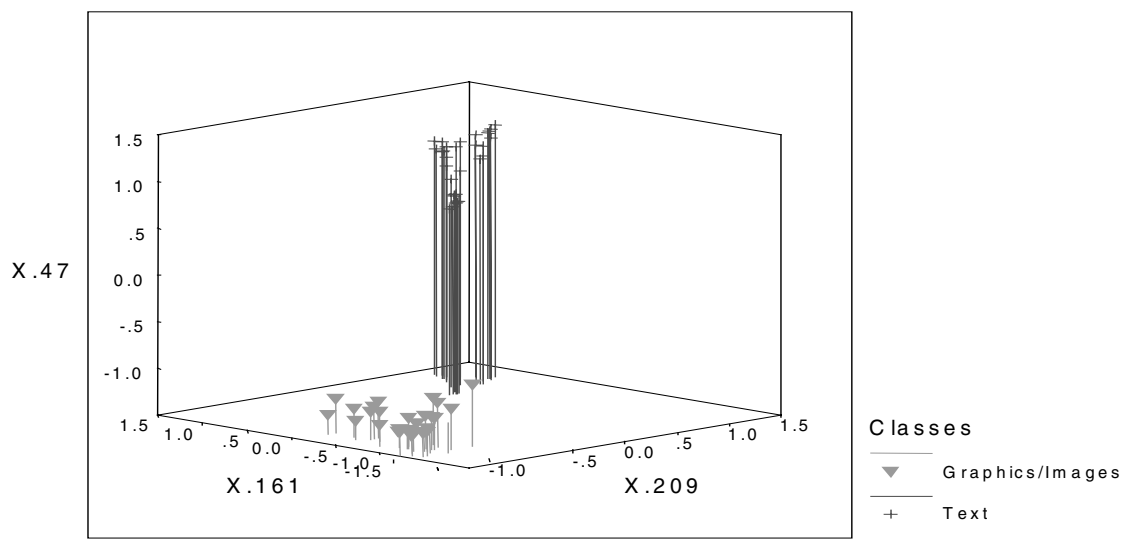

Fig. 4. Dispersion diagram of text and graphics/image pattern versus the three most discriminant features.

In figure 4 one can observe the excellent discriminant quality of the selected spectrum coefficients $\mathrm{x}_{161}, \mathrm{x}_{47}$ and $\mathrm{x}_{209}$, which makes the design of the automatic classifier a trivial task. Consequently, we have chosen a very simple three-layer perceptron-like neural network with a single inner layer formed by two neurons, which is displayed in figure 5 . 
Class 1 (text)
Class 2

(graphics/images)

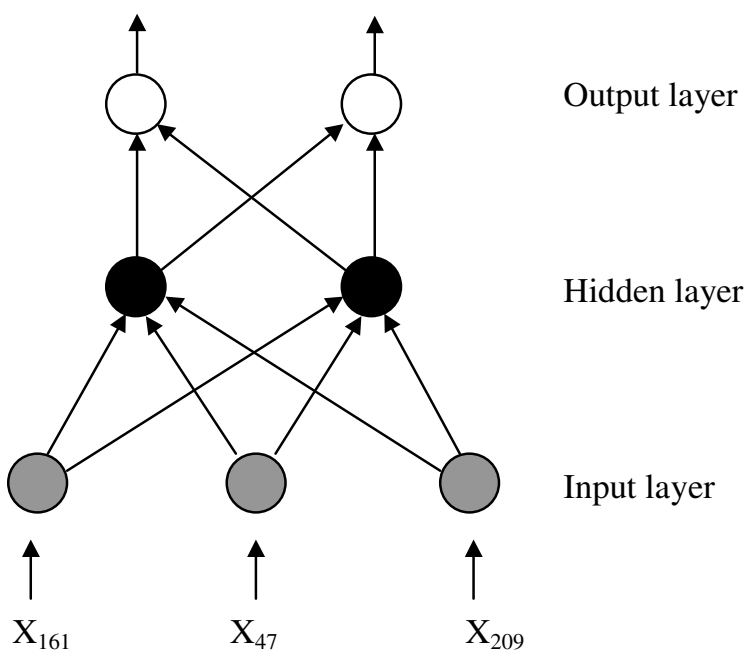

Fig. 5. Multilayer perceptron for text and graphics/images segmentation.

This neural network has been trained with the well-known backpropagation rule with a learning rate of 0.5 and a maximum permitted deviation of 0.1 . The neural network converges after very few iterations.

For testing we have employed 585 samples (297 for text regions and 288 for graphics/images regions) taken from a variety of documents: newspapers, magazines, books, etc. The results are summarized in the confusion matrix of table I, where Class 1 corresponds to text regions and Class 2 to graphics/images regions. The success ratios for both classes are always higher than 99 per cent.

Table 1. Confusion matrix for the 585 test samples.

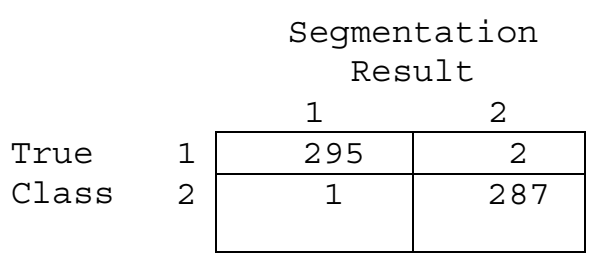

In figure 6 two examples of segmented documents are shown. The segmentation results appear on the right, where the windows stand for the image regions classified as text. 

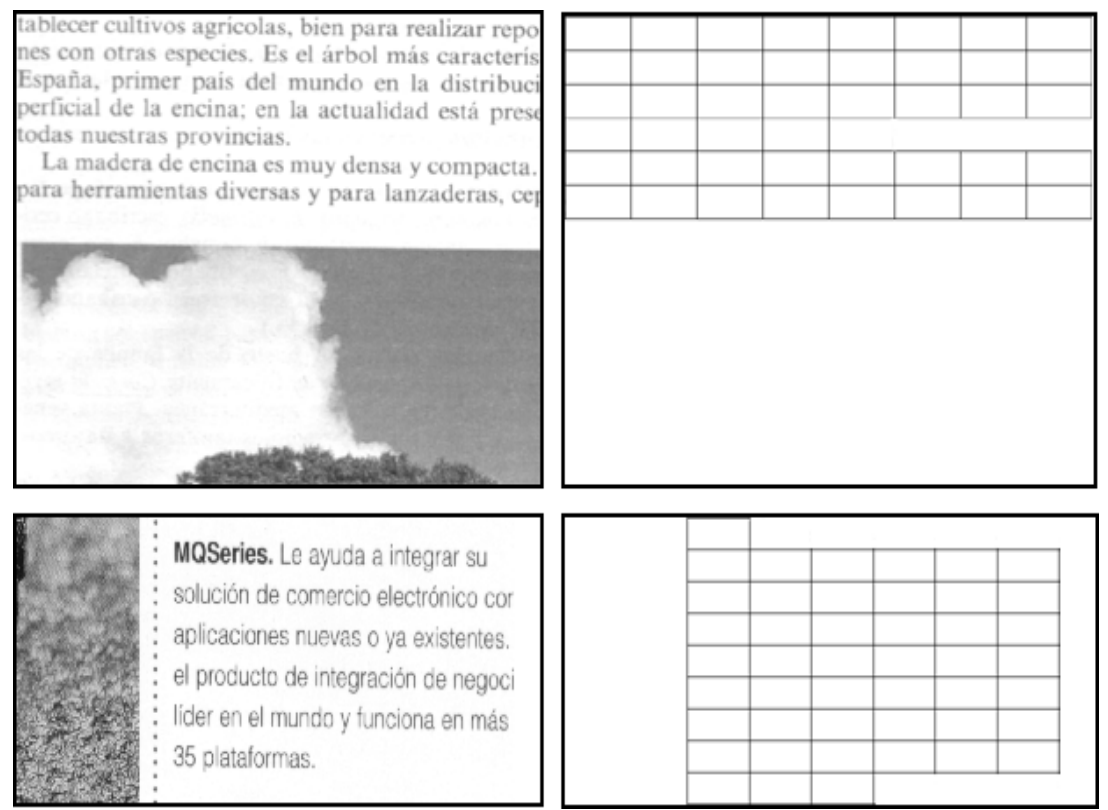

Fig. 6. Segmentation results. On the left are displayed the digitized documents. The automatic recognizer explores the document from top to bottom and from left to right with a $85 \times 35$ window. The segmentation results appear on the right, where the rectangles correspond to the text regions.

\section{Conclusions}

A novel, robust method for the segmentation of text and graphics/images in digitized documents has been described. This method is independent of fonts and type of document: books, magazines, newspapers, etc.

The basic idea is to use the magnitude spectrum of the gray level histogram of an image window as the initial set of discriminant features. Afterwards, a procedure for the automatic selection of the best discriminant features is applied. Due to the outstanding discriminant quality and the small number of selected features, the design of the classifier is quite trivial. A simple three-layer perceptron neural network, trained with the backpropagation rule, is applied for the segmentation of text and graphics/images, giving excellent results with at least $99 \%$ success. 


\section{References}

[1] H. Bunke, P.S.P. Wang, Handbook of Character Recognition and Document Image Analysis, World Scientific (1997).

[2] Z. Jiang, J. Liu, J. Liu Jin "SegChar: a tool for automatic text/graphics segmentation of engineering drawing images", Journal of Software Vol. 10 No. 6 (June 1999) 589-594.

[3] Z. Jiang, J. Liu, "A shielding method for segmentation of graphics touching text in engineering drawings", Porceedings of the SPIE. The International Society for Optical Engineering, Vol. 3305 (1998) 53-60.

[4] K. Tombre, C. Ah Soon, P. Dosch, A. Habed,, G. Masini, "Stable, robust and off-the-shelf methods for graphics recognition", Proceedings. Fourteenth Conference on Pattern Recognition. IEEE Comput. Soc, Vol. 1 (1998) 406-408.

[5] E. Martí, “Análisis de elementos gráficos en documentos”, RECV - Revista Electrónica de Visión por Computador, Centro de Visión por Computador, No. 0, (Octubre 1999). http://www.cvc.uab.es/recv/revista/000/00001-tut.htm.

[6] T. Ojala, M. Pietikäinen, and J. Nisula, "Determining composition of grain mixtures by texture classification based on feature distributions", Int. Journal of Pattern Recognition and Artificial Intelligence Vol. 10 No. 1 (1996) 73-82.

[7] T. Ojala, M. Pietikäinen, and D. Harwood, "Performance evaluation of texture measures with classification based on Kullback discrimination of distributions" Proc. $12^{\text {th }}$. Int. Conf. On Pattern Recognition, vol. 1, Jerusalem, Israel, (1994) 582-585.

[8] M. Unser, "Sum and difference histograms for texture classification", IEEE Trans. on Pattern Anal. and Machine Intell. 8 (1986) 118-125.

[9] A. L. Vickers and J.W. Modestino, "A maximum likelihood aproach to texture classification”, IEEE Trans. on Pattern Anal. and Machine Intell. 4 (1982) 61-68. 